\title{
Remarks on $\mathrm{N}=4$ Superconformal Extension of the Calogero Model
}

\author{
Anton V. Galajinsky* \\ Laboratory of Mathematical Physics, Tomsk Polytechnic University, \\ 634050 Tomsk, Lenin Ave. 30, Russian Federation \\ and \\ INFN-Laboratori Nazionali di Frascati, C.P. 13, 00044 Frascati, Italy
}

\begin{abstract}
Recently it was conjectured by Gibbons and Townsend that the large $n$ limit of an $N=4$ superconformal extension of the $n$-particle Calogero model might provide a microscopic description of the extreme Reissner-Nordström black hole near the horizon. In this paper a possibility to construct an $S U(1,1 \mid 2)$ invariant extension of the Calogero model is considered. We treat in detail the two-particle case and comment on some peculiarities intrinsic to $n>2$ generalizations.
\end{abstract}

PACS: 04.60.Ds; 11.30.Pb

Keywords: Calogero model, black holes, $N=4$ superconformal invariance

*galajin@mph.phtd.tpu.edu.ru, galajins@lnf.inf.it 
The Calogero model [1] has many interesting physical applications. It provides a nontrivial example of $n$-particle integrable system in one dimension (see Ref. [2] for a review). Calogero-type models are related to semisimple Lie algebras [2] and reveal an intriguing connection with Hamiltonian reductions of the $2 d$ Yang-Mills theory [3]. Higher dimensional generalizations appear in the context of fractional statistics [4] and higher spin gauge theories [5]. In quantum area the one-dimensional system turns out to be completely solvable (see e.g. Ref. [5] and references therein).

Quite recently the $n$-particle Calogero model has been brought to focus again, this time in the context of black hole physics [6]. It has been known for a long time [7] that the $n$-particle Calogero model with the Hamiltonian

$$
H=\frac{1}{2} \sum_{i=1}^{n} p_{i}^{2}+\frac{1}{2} \sum_{i<j} \frac{g^{2}}{\left(x_{i}-x_{j}\right)^{2}}
$$

where $g$ is a dimensionless coupling constant, exhibits the conformal invariance. The charges

$$
D=t H-\frac{1}{2} \sum_{i=1}^{n} x_{i} p_{i}, \quad K=t^{2} H-t \sum_{i=1}^{n} x_{i} p_{i}+\frac{1}{2} \sum_{i=1}^{n} x_{i} x_{i},
$$

are conserved and together with the Hamiltonian (1) form the so(1,2) algebra

$$
\{H, D\}=H, \quad\{H, K\}=2 D, \quad\{D, K\}=K,
$$

which is the conformal algebra in one dimension. The conjecture [6] that the $n$-particle Calogero model might be relevant for a microscopic description of the extreme ReissnerNordström black hole, at least near the horizon, originated from the fact that the near horizon geometry for this case has the $S U(1,1 \mid 2)$ isometry group, the bosonic subgroup being $S O(1,2)$. Taking into account that the extreme Reissner-Nordström black hole can be viewed as the configuration of four intersecting supergravity $D 3$-branes wrapped on $T^{6}[8]$ and assuming that each of the supergravity $D 3$-branes can be interpreted as a large number of coinciding microscopic D3-branes [6], one comes to the conclusion that there must be an $S U(1,1 \mid 2)$ invariant mechanics which governs the fluctuation of the branes in the region of the intersection [6]. Being conformally invariant, the $n$-particle Calogero system provides a possible candidate $^{1}$ for the bosonic part of such an $N=4$ superconformal mechanics.

In this brief note we discuss a possibility to construct an $N=4$ superconformal extension of the Calogero model. We treat in detail the two-particle case and comment on some peculiarities intrinsic to $n>2$ generalizations. Throughout the paper we choose to work in the Hamiltonian formalism.

As the first step one has to decide on the number of fermions to be assigned to each particle. Because the system must accommodate an $N=4$ supersymmetry and since in the one-particle limit one expects to reproduce the $N=4$ superconformal mechanics [9] it seems natural to append a pair of complex fermions $\left(\psi_{i}\right)^{*}=\bar{\psi}_{i}, i=1,2$, obeying the bracket $\left\{\psi_{i}, \bar{\psi}_{j}\right\}=-i \delta_{i j}$, to each bosonic canonical pair $\left(x_{1}, p_{1}\right)$ and $\left(x_{2}, p_{2}\right)$. Let us mark

\footnotetext{
${ }^{1}$ In the context of black hole physics the large $n$ limit is to follow [6].
} 
the fermionic degrees of freedom corresponding to each particle by the superscripts (1) and (2), respectively ${ }^{2}$. Apart from the conformal generators $H, D, K$, the $s u(1,1 \mid 2)$ superalgebra includes two complex supersymmetry charges $G_{1}, G_{2}$ (their conjugates will be denoted by $\left.\bar{G}_{1}, \bar{G}_{2}\right)$, the superconformal partners $S_{1}, S_{2}, \bar{S}_{1}, \bar{S}_{2}$, and the $R$-symmetry $s u(2)$ generators $J_{+}, J_{-}, J_{3}$ (in the basis chosen the commutation relations of the $s u(1,1 \mid 2)$ superalgebra are given in Appendix). As the bracket $\left\{G_{i}, \bar{G}_{j}\right\}=-2 i H \delta_{i j}, i, j=1,2$, makes part of the superalgebra, the contributions to $G_{i}, \bar{G}_{i}$ which are linear in the fermions should be adjusted so as to produce the Hamiltonian (1) (with $n=2$ for the case at hand). This is typical of supersymmetric (quantum) mechanics. Another important point to notice is a representation of the $s u(2)$ subalgebra. This is not unique and normally one chooses a "direct sum" representation (in this respect see also Refs. [10],[11])

$$
\begin{aligned}
& J_{+}=-i \psi_{1}^{(1)} \bar{\psi}_{2}^{(1)}-i \psi_{1}^{(2)} \bar{\psi}_{2}^{(2)}, \quad J_{-}=i \psi_{2}^{(1)} \bar{\psi}_{1}^{(1)}+i \psi_{2}^{(2)} \bar{\psi}_{1}^{(2)}, \\
& J_{3}=\frac{1}{2}\left(\psi_{1}^{(1)} \bar{\psi}_{1}^{(1)}-\psi_{2}^{(1)} \bar{\psi}_{2}^{(1)}+\psi_{1}^{(2)} \bar{\psi}_{1}^{(2)}-\psi_{2}^{(2)} \bar{\psi}_{2}^{(2)}\right),
\end{aligned}
$$

which implies that the fermions associated with each particle form a separate $S U(2)$ doublet. Because the supersymmetry generators transform under the action of $S U(2)$ (the brackets are given in the basis chosen)

$$
\begin{aligned}
& \left\{G_{1}, J_{+}\right\}=0, \quad\left\{G_{1}, J_{-}\right\}=\bar{G}_{2}, \quad\left\{G_{1}, J_{3}\right\}=\frac{i}{2} G_{1}, \\
& \left\{G_{2}, J_{+}\right\}=0, \quad\left\{G_{2}, J_{-}\right\}=-\bar{G}_{1}, \quad\left\{G_{2}, J_{3}\right\}=\frac{i}{2} G_{2},
\end{aligned}
$$

the structure of the fermionic contributions in $G_{1}$ and $G_{2}$ is completely fixed by the latter relations and the representation (4). As to the bosonic coefficients in front of the fermions, the fact that $G_{1}$ is nilpotent suggests a passage to the center of mass and relative coordinates $\left(x_{1}+x_{2}, p_{1}+p_{2}\right),\left(x_{1}-x_{2}, p_{1}-p_{2}\right)$. After adjusting the coefficients in a proper way one finds the following representation for the $N=4$ supersymmetry generators

$$
\begin{aligned}
G_{1}= & \frac{1}{\sqrt{2}}\left(p_{1}-p_{2}\right) \psi_{1}^{(1)}+\frac{g}{x_{1}-x_{2}} \bar{\psi}_{2}^{(1)}+\frac{2 i}{\sqrt{2}} \frac{1}{x_{1}-x_{2}} \psi_{1}^{(1)} \psi_{2}^{(1)} \bar{\psi}_{2}^{(1)}+ \\
& +\frac{1}{\sqrt{2}}\left(p_{1}+p_{2}\right) \psi_{1}^{(2)}+\frac{2 i}{\sqrt{2}} \frac{1}{x_{1}+x_{2}} \psi_{1}^{(2)} \psi_{2}^{(2)} \bar{\psi}_{2}^{(2)}, \\
G_{2}= & -\frac{1}{\sqrt{2}}\left(p_{1}-p_{2}\right) \bar{\psi}_{2}^{(1)}+\frac{g}{x_{1}-x_{2}} \psi_{1}^{(1)}+\frac{2 i}{\sqrt{2}} \frac{1}{x_{1}-x_{2}} \psi_{1}^{(1)} \bar{\psi}_{1}^{(1)} \bar{\psi}_{2}^{(1)}- \\
& -\frac{1}{\sqrt{2}}\left(p_{1}+p_{2}\right) \bar{\psi}_{2}^{(2)}+\frac{2 i}{\sqrt{2}} \frac{1}{x_{1}+x_{2}} \psi_{1}^{(2)} \bar{\psi}_{1}^{(2)} \bar{\psi}_{2}^{(2)},
\end{aligned}
$$

which yield the Hamiltonian

$$
\begin{aligned}
H= & \frac{1}{2} p_{1}^{2}+\frac{1}{2} p_{2}^{2}+\frac{g^{2}}{2\left(x_{1}-x_{2}\right)^{2}}+\frac{2 i g}{\sqrt{2}\left(x_{1}-x_{2}\right)^{2}} \psi_{1}^{(1)} \psi_{2}^{(1)}+\frac{2 i g}{\sqrt{2}\left(x_{1}-x_{2}\right)^{2}} \bar{\psi}_{1}^{(1)} \bar{\psi}_{2}^{(1)}+ \\
& +\frac{2}{\left(x_{1}-x_{2}\right)^{2}} \psi_{1}^{(1)} \bar{\psi}_{1}^{(1)} \psi_{2}^{(1)} \bar{\psi}_{2}^{(1)}+\frac{2}{\left(x_{1}+x_{2}\right)^{2}} \psi_{1}^{(2)} \bar{\psi}_{1}^{(2)} \psi_{2}^{(2)} \bar{\psi}_{2}^{(2)}
\end{aligned}
$$

\footnotetext{
${ }^{2}$ In order to make the formulae more readable we loosely put the index $i$ on both $\psi$ and $\bar{\psi}$ down. A more proper notation would be $\left(\psi^{i}\right)^{*}=\bar{\psi}_{i},\left\{\psi^{i}, \bar{\psi}_{j}\right\}=-i \delta^{i}{ }_{j}$.
} 
Because the pairs $G_{1}, \bar{G}_{1}$ and $G_{2}, \bar{G}_{2}$ mutually commute, the conservation in time of these generators is guaranteed by Jacobi identities.

To construct a representation for the superconformal generators $S_{1}, \bar{S}_{1}, S_{2}, \bar{S}_{2}$ one appeals to the $s u(1,1 \mid 2)$ superalgebra (see Appendix). It suffices to calculate the Poisson bracket of the supersymmetry charges with the generator of special conformal transformations $K$ (see Eq. (2) above with $H$ taken from Eq. (7)) which gives

$$
\begin{aligned}
& S_{1}=t G_{1}-\frac{1}{\sqrt{2}}\left(x_{1}-x_{2}\right) \psi_{1}^{(1)}-\frac{1}{\sqrt{2}}\left(x_{1}+x_{2}\right) \psi_{1}^{(2)}, \\
& S_{2}=t G_{2}+\frac{1}{\sqrt{2}}\left(x_{1}-x_{2}\right) \bar{\psi}_{2}^{(1)}+\frac{1}{\sqrt{2}}\left(x_{1}+x_{2}\right) \bar{\psi}_{2}^{(2)} .
\end{aligned}
$$

It is straightforward to check the the full algebra is closed (the commutation relations are gathered in Appendix) and we conclude that the Hamiltonian (7) governs the dynamics of an $S U(1,1 \mid 2)$ invariant extension of the two-particle Calogero model. Notice that in the one-particle limit (i.e. setting $x_{2}=0, p_{2}=0, \psi_{i}^{(2)}=0$ ) the system (7) reproduces the $N=4$ superconformal mechanics of Ref. [9] (with the parameters $c=0, f=1$; see [9] for more details). A few comments are in order.

i) Let us compare our result with the previous attempts [10],[11] to construct an $N=4$ superconformal extension of the Calogero model. According to the analysis of Ref. [10] for generic values of the coupling constant $g$ and for $n>2$ there is no way to build an $S U(1,1 \mid 2)$ invariant extension, while for $n=2$ this can be done only for a particular value of the coupling constant (see Ref. [10] for more details). As we have seen above, taking a more general ansatz for the supersymmetry generators one can weaken the restriction on the coupling constant and construct a two-particle model for generic values of the coupling constant ${ }^{3}$. In Ref. [11] an $S U(1,1 \mid 2)$-invariant model in two dimensions has been constructed without any restrictions on the coupling constant or the number of particles. Since a naive dimensional reduction to one dimension does not preserve all four supersymmetries it seems interesting to search for a more sophisticated reduction.

ii) Although we did not try to systematically extend our analysis to the $n>2$ case, a preliminary consideration shows that this may require a modification of the $s u(2)$ representation (4). One such possibility is to mix fermions in the $s u(2)$-generators so that the fermions belonging to different particles share the same $S U(2)$ spinor representation. For example, for the three-particle case the generators

$$
\begin{aligned}
& J_{+}=\frac{1}{\gamma}[-i \psi_{1}^{(1)} \bar{\psi}_{2}^{(1)}-i \psi_{1}^{(2)} \bar{\psi}_{2}^{(2)}-i \psi_{1}^{(3)} \bar{\psi}_{2}^{(3)}+\lambda\left(\psi_{1}^{(1)} \bar{\psi}_{2}^{(2)}+\psi_{1}^{(2)} \bar{\psi}_{2}^{(1)}+\psi_{1}^{(1)} \bar{\psi}_{2}^{(3)}+\psi_{1}^{(3)} \bar{\psi}_{2}^{(1)}+\right. \\
&\left.\left.\quad+\psi_{1}^{(3)} \bar{\psi}_{2}^{(2)}+\psi_{1}^{(2)} \bar{\psi}_{2}^{(3)}\right)\right] \\
& J_{-}=\frac{1}{\bar{\gamma}}\left[i \psi_{2}^{(1)} \bar{\psi}_{1}^{(1)}+i \psi_{2}^{(2)} \bar{\psi}_{1}^{(2)}+i \psi_{2}^{(3)} \bar{\psi}_{1}^{(3)}+\bar{\lambda}\left(\psi_{2}^{(2)} \bar{\psi}_{1}^{(1)}+\psi_{2}^{(1)} \bar{\psi}_{1}^{(2)}+\psi_{2}^{(3)} \bar{\psi}_{1}^{(1)}+\psi_{2}^{(1)} \bar{\psi}_{1}^{(3)}+\right.\right.
\end{aligned}
$$

\footnotetext{
${ }^{3} \mathrm{~A}$ passage to the center of mass and relative coordinates in the Hamiltonian of the two-particle Calogero model brings it to the sum of a free particle and the conformal mechanics [12], both admitting an $N=4$ superconformal extension. Thus, the existence of an $S U(1,1 \mid 2)$ invariant extension of the two-particle Calogero model for an arbitrary value of the coupling constant might be anticipated on this general ground. We thank Sergey Krivonos for pointing this out to us.
} 


$$
\begin{gathered}
\left.\left.+\psi_{2}^{(2)} \bar{\psi}_{1}^{(3)}+\psi_{2}^{(3)} \bar{\psi}_{1}^{(2)}\right)\right] \\
J_{3}=\frac{1}{2}\left[\psi_{1}^{(1)} \bar{\psi}_{1}^{(1)}-\psi_{2}^{(1)} \bar{\psi}_{2}^{(1)}+\psi_{1}^{(2)} \bar{\psi}_{1}^{(2)}-\psi_{2}^{(2)} \bar{\psi}_{2}^{(2)}+\psi_{1}^{(3)} \bar{\psi}_{1}^{(3)}-\psi_{2}^{(3)} \bar{\psi}_{2}^{(3)}\right]
\end{gathered}
$$

form the $s u(2)$ algebra, provided $\lambda-\bar{\lambda}-i \lambda \bar{\lambda}=0,1+2 \lambda \bar{\lambda}=\gamma \bar{\gamma}$, the simplest solution being $\lambda=2 i, \gamma=3$.

iii) A representation of the $s u(1,1 \mid 2)$ algebra constructed in this paper involves a central charge which appears in the brackets of the supersymmetry generators with the superconformal ones and is proportional to the (dimensionless) coupling constant $g$ (see Appendix). If one assumes that $n$-particle $S U(1,1 \mid 2)$-invariant model can be constructed along similar lines, the central term is likely to have the form $n(n-1) g$ (in this respect see also Ref. [11]). Then, in order to preserve the algebraic structure, the large $n$ limit is to be accompanied by taking $g$ to be small with $n(n-1) g$ fixed.

\section{Acknowledgements}

We thank Pijush K. Ghosh and Sergey Krivonos for useful discussions. This work was partially supported by the Ministry of Education of Russian Federation, grant E02-2.0-7, and NATO Collaborative Linkage grant PST.CLG. 979389.

\section{Appendix}

In this appendix we expose the Poisson brackets of the generators of the $s u(1,1 \mid 2)$ superalgebra in the basis chosen (vanishing brackets are omitted)

$$
\begin{aligned}
& \left\{G_{1}, \bar{G}_{1}\right\}=-2 i H, \quad\left\{G_{2}, \bar{G}_{2}\right\}=-2 i H, \quad\left\{G_{1}, J_{-}\right\}=\bar{G}_{2}, \quad\left\{G_{1}, J_{3}\right\}=\frac{i}{2} G_{1}, \\
& \left\{\bar{G}_{1}, J_{+}\right\}=G_{2}, \quad\left\{\bar{G}_{1}, J_{3}\right\}=-\frac{i}{2} \bar{G}_{1}, \quad\left\{G_{2}, J_{-}\right\}=-\bar{G}_{1}, \quad\left\{G_{2}, J_{3}\right\}=\frac{i}{2} G_{2}, \\
& \left\{\bar{G}_{2}, J_{+}\right\}=-G_{1}, \quad\left\{\bar{G}_{2}, J_{3}\right\}=-\frac{i}{2} \bar{G}_{2}, \quad\left\{D, G_{1}\right\}=-\frac{1}{2} G_{1}, \quad\left\{D, G_{2}\right\}=-\frac{1}{2} G_{2}, \\
& \left\{D, \bar{G}_{1}\right\}=-\frac{1}{2} \bar{G}_{1}, \quad\left\{D, \bar{G}_{2}\right\}=-\frac{1}{2} \bar{G}_{2}, \quad\left\{K, G_{1}\right\}=-S_{1}, \quad\left\{K, G_{2}\right\}=-S_{2}, \\
& \left\{K, \bar{G}_{1}\right\}=-\bar{S}_{1}, \quad\left\{K, \bar{G}_{2}\right\}=-\bar{S}_{2}, \quad\left\{G_{1}, S_{2}\right\}=-2 i J_{+}, \quad\left\{G_{1}, \bar{S}_{1}\right\}=-2 i D+2 J_{3}, \\
& \left\{G_{1}, \bar{S}_{2}\right\}=-\frac{i}{\sqrt{2}} g, \quad\left\{G_{2}, S_{1}\right\}=2 i J_{+}, \quad\left\{G_{2}, \bar{S}_{1}\right\}=\frac{i}{\sqrt{2}} g, \quad\left\{G_{2}, \bar{S}_{2}\right\}=-2 i D+2 J_{3}, \\
& \left\{\bar{G}_{1}, S_{1}\right\}=-2 i D-2 J_{3}, \quad\left\{\bar{G}_{1}, S_{2}\right\}=-\frac{i}{\sqrt{2}} g, \quad\left\{\bar{G}_{1}, \bar{S}_{2}\right\}=-2 i J_{-}, \quad\left\{\bar{G}_{2}, S_{1}\right\}=\frac{i}{\sqrt{2}} g, \\
& \left\{\bar{G}_{2}, S_{2}\right\}=-2 i D-2 J_{3}, \quad\left\{\bar{G}_{2}, \bar{S}_{1}\right\}=2 i J_{-}, \quad\left\{S_{1}, \bar{S}_{1}\right\}=-2 i K, \quad\left\{S_{2}, \bar{S}_{2}\right\}=-2 i K, \\
& \left\{S_{1}, J_{-}\right\}=\bar{S}_{2}, \quad\left\{S_{1}, J_{3}\right\}=\frac{i}{2} S_{1}, \quad\left\{S_{2}, J_{-}\right\}=-\bar{S}_{1}, \quad\left\{S_{2}, J_{3}\right\}=\frac{i}{2} S_{2}, \quad\left\{\bar{S}_{1}, J_{+}\right\}=S_{2}, \\
& \left\{\bar{S}_{1}, J_{3}\right\}=-\frac{i}{2} \bar{S}_{1}, \quad\left\{\bar{S}_{2}, J_{+}\right\}=-S_{1}, \quad\left\{\bar{S}_{2}, J_{3}\right\}=-\frac{i}{2} \bar{S}_{2}, \quad\left\{D, S_{1}\right\}=\frac{1}{2} S_{1}, \\
& \left\{D, S_{2}\right\}=\frac{1}{2} S_{2}, \quad\left\{D, \bar{S}_{1}\right\}=\frac{1}{2} \bar{S}_{1}, \quad\left\{D, \bar{S}_{2}\right\}=\frac{1}{2} \bar{S}_{2}, \quad\left\{H, S_{1}\right\}=G_{1}, \quad\left\{H, S_{2}\right\}=G_{2}, \\
& \left\{H, \bar{S}_{1}\right\}=\bar{G}_{1}, \quad\left\{H, \bar{S}_{2}\right\}=\bar{G}_{2}, \quad\{H, D\}=H_{1}, \quad\{H, K\}=2 D, \quad\{D, K\}=K, \\
& \left\{J_{+}, J_{-}\right\}=-2 i J_{3}, \quad\left\{J_{+}, J_{3}\right\}=i J_{+}, \quad\left\{J_{-}, J_{3}\right\}=-i J_{-} . \quad
\end{aligned}
$$




\section{References}

[1] F. Calogero, J. Math. Phys. 10 (1969) 2197.

[2] M.A. Olshanetsky and A.M. Perelomov, Phys. Rep. 71 (1981) 313.

[3] A. Gorsky and N. Nekrasov, Nucl. Phys. B 414 (1994) 213.

[4] A. Polychronakos, Nucl. Phys. B 324 (1989) 597.

T.H. Hansson, J.M. Leinaas and J. Myrheim, Nucl. Phys. B 384 (1992) 559.

[5] L. Brink, T.H. Hansson and M.A. Vasiliev, Phys. Lett. B 286 (1992) 109.

[6] G.W. Gibbons and P.K. Townsend, Phys. Lett. B 454 (1999) 187.

[7] G. Barucchi and T. Regge, J. Math. Phys. 18 (1977) 1149.

S. Wojciechowski, Phys. Lett. A 64 (1977) 273.

[8] I.R. Klebanov and A.A. Tseytlin, Nucl. Phys. B 475 (1996) 179.

V. Balasubramanian and F. Larsen, Nucl. Phys. B 495 (1997) 206.

[9] E. Ivanov, S. Krivonos and V. Leviant, J. Phys. A: Math. Gen. 22 (1989) 4201.

[10] N. Wyllard, J. Math. Phys. 41 (2000) 2826.

[11] P.K. Ghosh, J. Phys. A 34 (2001) 5583.

[12] V. De Alfaro, S. Fubini and G. Furlan, Nuovo Cim. A 34 (1974) 569. 